\title{
Properties of In-Doped ZnO Films Grown by Metalorganic Chemical Vapor Deposition on GaN(0001) Templates
}

\author{
TAMMY BEN-YAACOV,${ }^{1,3}$ TOMMY IVE ${ }^{1}$ CHRIS G. VAN DE WALLE,${ }^{1}$ \\ UMESH K. MISHRA, ${ }^{2}$ JAMES S. SPECK, ${ }^{1}$ and STEVEN P. DENBAARS ${ }^{1,2}$ \\ 1.-Materials Department, University of California, Santa Barbara, CA 93106, USA. 2.-Elec- \\ trical and Computer Engineering Department, University of California, Santa Barbara, CA 93106, \\ USA. 3.—e-mail: tammy@umail.ucsb.edu
}

We investigated the properties of indium-doped zinc oxide layers grown by metalorganic chemical vapor deposition on semi-insulating GaN(0001) templates. Specular and transparent films were grown with $n$-type carrier concentrations up to $1.82 \times 10^{19} \mathrm{~cm}^{-3}$ as determined by Hall measurements, and all In-doped films had carrier concentrations significantly higher than that of a comparable undoped film. For low In flows, the carrier concentration increased accordingly with trimethyl-indium (TMIn) flow until a maximum carrier concentration of $1.82 \times 10^{19} \mathrm{~cm}^{-3}$ was realized. For higher In flows, the carrier concentration decreased with increasing TMIn flow rate. Sheet resistance as low as $185 \Omega /$ sq was achieved for the In-doped films, which is a significant decrease from that of a comparable undoped $\mathrm{ZnO}$ film. Our $n$-type doping studies show that In is an effective dopant for controlling the $n$-type conductivity of $\mathrm{ZnO}$.

Key words: $\mathrm{ZnO}$, zinc oxide, doping, $n$-type, MOCVD, metalorganic chemical vapor deposition, $\mathrm{ZnO}$ :In, indium doping, electrical characterization, growth, crystal growth, thin films

$\mathrm{ZnO}$ has many unique electrical, piezoelectric, and optical properties ${ }^{1-3}$ which make it an applicable material for optoelectronic devices. For this reason, there is a widespread effort toward epitaxial growth of high-quality, single-crystal $\mathrm{ZnO}$ thin films, as well as much work on controlling the electrical properties of these films. $\mathrm{ZnO}$ has a wide direct bandgap of $3.4 \mathrm{eV}$, and it crystallizes in the wurtzite structure. It has an exciton binding energy of $60 \mathrm{meV},{ }^{4}$ and so it has the potential for stable and efficient optical emission at room temperature. The availability of high-quality bulk $\mathrm{ZnO}$ substrates opens the possibility for homoepitaxial growth. These properties make $\mathrm{ZnO}$ an excellent candidate for various device applications.

For $\mathrm{ZnO}$ to be used for optoelectronic applications, it is necessary to control the $n$-type conductivity of $\mathrm{ZnO}$ films, while simultaneously achieving good

(Received August 1, 2009; accepted November 19, 2009; published online December 11, 2009) surface morphology and crystal quality. This is a major challenge, as undoped $\mathrm{ZnO}$ almost always exhibits $n$-type conductivity, and there is a significant body of work toward identifying the cause of high unintentional conductivity (see Refs. 5-7 and references therein). In addition, the introduction of dopants has a strong impact on film morphology and crystal quality.

For II-VI compound semiconductors including $\mathrm{ZnO}$, group III elements, including $\mathrm{In}, \mathrm{Ga}$, and $\mathrm{Al}$, are regarded as ideal donors due to their valence. ${ }^{8,9}$ Most efforts reported on $n$-type doping of $\mathrm{ZnO}$ are for transparent contact applications, with $\mathrm{Ga}$ as the dopant. It has been reported that thin $\mathrm{ZnO}$ :Ga layers work well as a contact to $p-\mathrm{GaN}$ in InGaN lightemitting diodes (LEDs), and that they improve the light extraction efficiency of the devices. ${ }^{10}$ We have grown such structures and also realized promising results with thin $\mathrm{ZnO}: \mathrm{Ga}$ layers as contacts to InGaN LEDs. ${ }^{11}$ Little work has been reported, however, on controlled $n$-type doping of thicker single-crystal $\mathrm{ZnO}$ films. 
There is a lot of effort toward fabricating highquality $\mathrm{ZnO}$ contacts with high conductivity, low resistivity, and high transparency in nitride-based devices such as LEDs and solar cells. Thin-film metals have been widely studied as contact materials to $p$-type $\mathrm{GaN}, \mathrm{Ni} / \mathrm{Au}$ being the most common. The efficiency of these devices is partly limited by absorption in the semi-transparent $p$-contact $\mathrm{Ni} / \mathrm{Au}$ layer, and therefore it is of interest to implement transparent conducting oxides (TCO) as an alternative contact scheme. ${ }^{12}$ The dominant TCO is amorphous indium tin oxide (ITO), most commonly deposited by sputtering techniques. ${ }^{13-16}$ Due to the deposition technique and material quality, it has been shown that there is usually a high contact resistance at the $p$-GaN/ITO interface, and consequently ITO-contacted LEDs exhibited a significantly higher turn-on voltage than that of metalcontacted devices. ${ }^{13} \mathrm{ZnO}$ has the potential to be a more favorable candidate than ITO in this regard, because the $a$-axis lattice constant of $\mathrm{ZnO}$ is closely matched to that of $\mathrm{GaN}$, and so high-quality $\mathrm{ZnO}$ films can be grown epitaxially on GaN. ${ }^{17,18}$ There is also great economical motivation for implementing $\mathrm{ZnO}$ as a contact over ITO, as the price of In has been rising and is significantly higher than the price of $\mathrm{Zn}$.

The ability to control the $n$-type conductivity of high-quality $\mathrm{ZnO}$ films while sustaining good crystal quality and surface morphology is invaluable for device applications. In this study, we present the properties of $0.5-\mu \mathrm{m}$-thick In-doped $\mathrm{ZnO}(0001)$ films grown by metalorganic chemical vapor deposition (MOCVD) on Fe-doped, semi-insulating GaN(0001) templates.

A Thomas Swan close-coupled showerhead MOCVD reactor was used for the growth experiments. Diethyl-zinc (DEZ), trimethyl-indium (TMIn), and pure $\mathrm{O}_{2}$ were the $\mathrm{Zn}$, In, and oxygen sources, respectively. Nitrogen was used as the carrier gas. $\mathrm{Fe}$-doped, semi-insulating $\mathrm{GaN} / \mathrm{Al}_{2} \mathrm{O}_{3}$ templates from LUMILOG were used as substrates for these growth experiments, which allowed for electrical isolation of the $\mathrm{ZnO}$ film.

An undoped $\mathrm{ZnO}$ buffer layer was deposited at $450^{\circ} \mathrm{C}$ with a reactor pressure of 50 torr before the temperature was increased to $950^{\circ} \mathrm{C}$ for the hightemperature deposition of the $\mathrm{ZnO}$ epitaxial layer. At $950^{\circ} \mathrm{C}$, a thin unintentionally doped (UID) $\mathrm{ZnO}$ layer was deposited first, and then In was introduced for the growth of a thicker $\mathrm{ZnO}$ :In layer. Figure 1a shows a diagram of the growth structure. The total thickness of the $\mathrm{ZnO}$ layers was $0.5 \mu \mathrm{m}$. The VI/II ratio was 550 and 11,000 for the buffer layer and the high-temperature epitaxial layer, respectively. The TMIn flows ranged from $3 \mathrm{sccm}$ to $50 \mathrm{sccm}$. The growth rate was $2.2 \mathrm{~A} / \mathrm{s}$. These growth conditions were realized by an extensive heteroepitaxial growth study of undoped $\mathrm{ZnO}$ films grown on $\mathrm{GaN}(0001)$ templates, which was conducted prior to the In-doping experiments. These growth conditions yielded UID $\mathrm{ZnO}$ layers with locally straight (a)

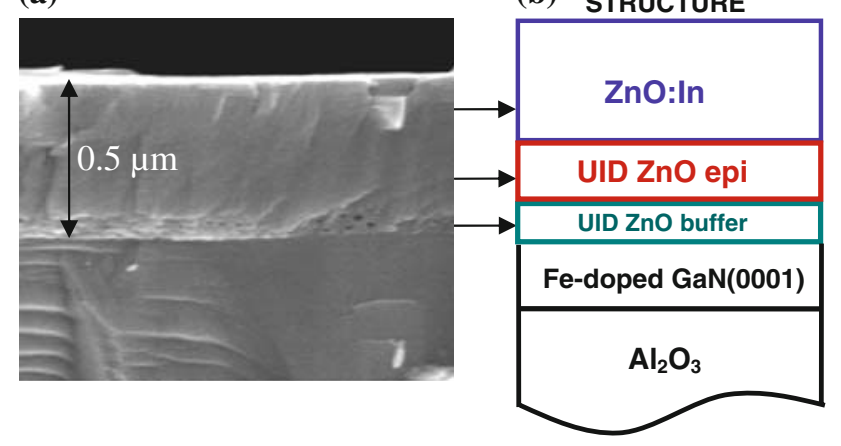

Fig. 1. (a) Cross-sectional SEM image of sample $C$ (Table I) with the layer structure outlined to the right. (b) Growth structure for $\mathrm{ZnO}$ In-doping experiments. The growth sequence on Fe-doped, semiinsulating GaN(0001) templates is as follows: (1) undoped $\mathrm{ZnO}$ buffer, (2) undoped $\mathrm{ZnO}$ epilayer, (3) In-doped $\mathrm{ZnO}$ epilayer. The epilayer growth temperature and reactor pressure were $950^{\circ} \mathrm{C}$ and 50 torr, respectively.

steps indicating step-flow growth, and a root-meansquare roughness less than $2.6 \mathrm{~nm} \quad(20 \mu \mathrm{m} \times$ $20 \mu \mathrm{m}$ ). The full-width at half-maximum (FWHM) across the symmetric (0002) $\mathrm{x}$-ray rocking curve was as narrow as $0.091^{\circ} .{ }^{19}$ We compared the electrical properties, the film and surface morphology, and the crystal quality of the In-doped films with a comparable undoped film to clearly see the effects of the In doping.

Figure 1a shows a cross-sectional scanning electron microscopy (SEM) image of a $\mathrm{ZnO}$ :In film doped with $5 \mathrm{sccm}$ TMIn, with the layer structure shown in Fig. 1b. In this image it is possible to clearly see the $\mathrm{ZnO} / \mathrm{GaN}$ interface, the buffer layer, and the epilayer. No interface was observed between the UID $\mathrm{ZnO}$ and the In-doped $\mathrm{ZnO}$, and In incorporation had negligible impact on the film morphology.

Table I shows the properties of both a UID ZnO film (sample A) and of the In-doped films grown in this study (samples B-K). Carrier concentration, mobility, and sheet resistance were evaluated by Hall measurements using the Van der Pauw configuration with In dots. All In-doped films had carrier concentrations significantly greater than that of the undoped sample, as the range of carrier concentration for In-doped samples was $5.3 \times$ $10^{18} \mathrm{~cm}^{-3}$ to $1.8 \times 10^{19} \mathrm{~cm}^{-3}$, as compared with $5.4 \times 10^{17} \mathrm{~cm}^{-3}$ for the undoped sample.

The sheet resistances of the In-doped $\mathrm{ZnO}$ films were significantly lower than that of the undoped sample. A minimum sheet resistance of $184 \Omega / \mathrm{sq}$ was achieved for sample $\mathrm{C}$, as compared with $3100 \Omega / \mathrm{sq}$ for the undoped sample. For comparison, the sheet resistances for 200 -nm-thick sputtered and e-beam-deposited ITO films have been reported to be as low as $15 \Omega /$ sq and $33 \Omega /$ sq, respectively. ${ }^{15}$ The $\mathrm{ZnO}$ :In film, which is more than double the thickness of these ITO films, has a sheet resistance greater by only a factor of about 6 . With some further improvement, In-doped $\mathrm{ZnO}$ will be able to compete with ITO in terms of resistivity. 
Table I. Summary of properties of the In-doped ZnO layers presented in this study

\begin{tabular}{|c|c|c|c|c|c|c|}
\hline Sample & TMIn Flow (sccm) & $n_{\text {Hall }}\left(\mathrm{cm}^{-3}\right)$ & {$[\operatorname{In}]\left(\mathrm{cm}^{-\mathbf{3}}\right)$} & $\mu\left(\mathrm{cm}^{2} / \mathrm{Vs}\right)$ & $R_{\text {sheet }}(\Omega /$ sq $)$ & $\Delta \omega(0002)($ degree $)$ \\
\hline A (undoped) & 0 & $5.4 \times 10^{17}$ & $1.4 \times 10^{18}$ & 37 & 3100 & 0.091 \\
\hline B & 3 & $5.32 \times 10^{18}$ & $1.0 \times 10^{19}$ & 38 & 622 & 0.136 \\
\hline $\mathrm{C}$ & 3.6 & $1.0 \times 10^{19}$ & - & 37 & 540 & - \\
\hline $\mathrm{D}$ & 4.1 & $1.36 \times 10^{19}$ & - & 37 & 348 & 0.146 \\
\hline $\mathrm{E}$ & 4.6 & $1.6 \times 10^{19}$ & - & 38 & 206 & - \\
\hline $\mathrm{F}$ & 5 & $1.82 \times 10^{19}$ & $3.7 \times 10^{19}$ & 37 & 184 & 0.153 \\
\hline $\mathrm{G}$ & 6 & $1.79 \times 10^{19}$ & $8.0 \times 10^{19}$ & 35 & 198 & 0.165 \\
\hline $\mathrm{H}$ & 12 & $1.39 \times 10^{19}$ & $1.3 \times 10^{20}$ & 35 & 262 & 0.177 \\
\hline $\mathrm{I}$ & 25 & $1.38 \times 10^{19}$ & $4.0 \times 10^{20}$ & 30 & 299 & 0.181 \\
\hline J & 35 & $1.12 \times 10^{19}$ & $5.5 \times 10^{20}$ & 45 & 245 & 0.182 \\
\hline $\mathrm{K}$ & 50 & $7.7 \times 10^{18}$ & $6.0 \times 10^{20}$ & 33 & 489 & - \\
\hline
\end{tabular}

Listed are donor concentration $\left(n_{\text {Hall }}\right)$, mobility $(\mu)$, and sheet resistance $\left(R_{\text {sheet }}\right)$ as determined by Hall measurements, the indium concentration ([In]) as determined by secondary-ion mass spectroscopy (SIMS), and the x-ray diffraction (XRD) FWHM $\Delta \omega$ of the $\mathrm{ZnO}(0002)$ reflection.

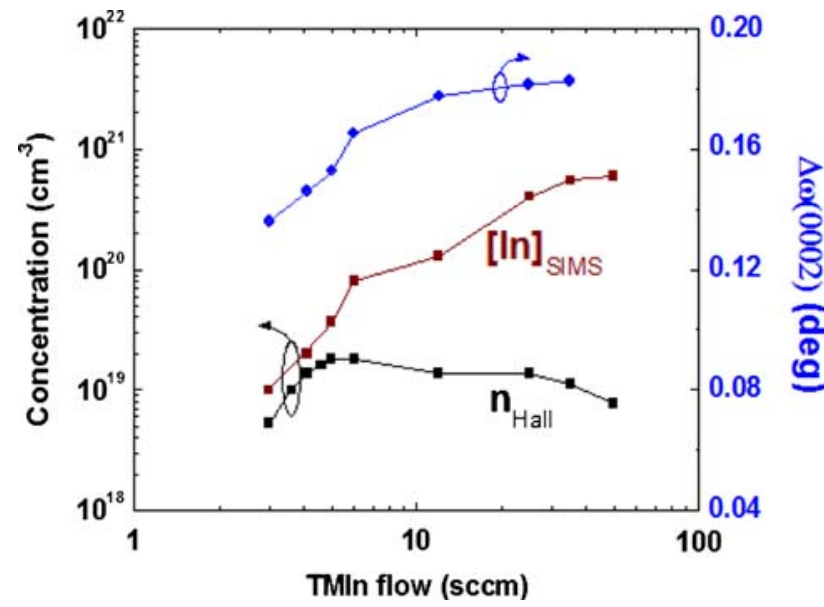

Fig. 2. Dependence of electron concentration (measured by Hall effect) and In concentration (measured with SIMS) on TMIn flow (left $y$-axis), and the FWHM $\Delta \omega$ of rocking curves for the (0002) reflection as a function of TMIn flow (right $y$-axis).

The dependence of electron concentration, as measured by Hall effect, and chemical In concentration, measured with secondary-ion mass spectroscopy (SIMS), on TMIn flow is shown in Fig. 2. For low In flows, the electron concentration increased as the dopant flow increased, in good agreement with the In concentration measured by SIMS. The highest electron concentration $(1.8 \times$ $10^{19} \mathrm{~cm}^{-3}$ ) was realized for sample $\mathrm{C}$. When the In concentration was increased beyond this concentration the measured electron concentration decreased, which may indicate that much of the In was not incorporated as a simple shallow donor.

The In concentration as measured by SIMS scaled roughly with TMIn flow and varied between $1 \times$ $10^{19} \mathrm{~cm}^{-3}$ and $6 \times 10^{20} \mathrm{~cm}^{-3}$. The samples with higher In concentrations were overdoped, i.e., the In concentration was so high that the material quality began to degrade. The crystal quality was assessed by recording the FWHM of XRD $\omega$-scans across the (a) Undoped $\mathrm{ZnO}$

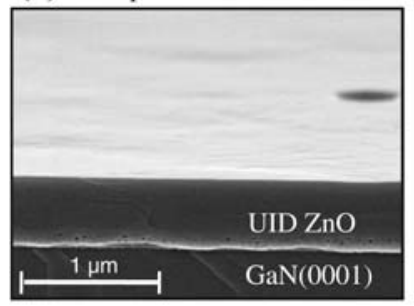

(c) 6 sccm TMIn

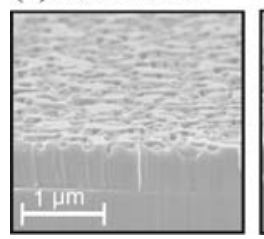

(d) $12 \mathrm{sccm}$ TMIn

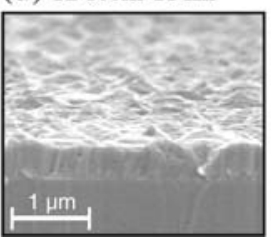

Fig. 3. SEM images of (a) a UID ZnO layer and (b-e) ZnO:In films grown on $\mathrm{GaN}(0001)$ templates, showing the effect of In doping on surface morphology. All ZnO:In films are homogeneous and compact, and surface morphology improves for lower In flows: (a) sample A; film is smooth, hexagonal pit can be observed; (b) sample B, (c) sample G, (d) sample $H$, and (e) sample I.

symmetric (0002) reflection. Table I summarizes the results from these measurements, and Fig. 2 also shows the dependence of FWHM $\Delta \omega$ of the rocking curves on the TMIn flow. The ZnO:In films have good crystal quality, as sample B had a narrow FWHM of $0.136^{\circ}$ (489 arcseconds), and it is clear that the quality of the $\mathrm{ZnO}$ :In films decreased with increasing TMIn flow.

SEM images were used to evaluate the effect of In on film and surface morphology. Figure 3 shows the undoped film (a), and four In-doped films (b-e) for a range of TMIn flows. The undoped $\mathrm{ZnO}$ film was very smooth with occasional hexagonal pits, as shown in Fig. 3a. As shown in Fig. 3b, the introduction of $3 \mathrm{sccm}$ of TMIn, which was in the low part of the doping range for this experimental series, had a noticeable impact on the surface morphology, which became slightly rougher. Figure 
(a) $0.06 \mathrm{sccm}$

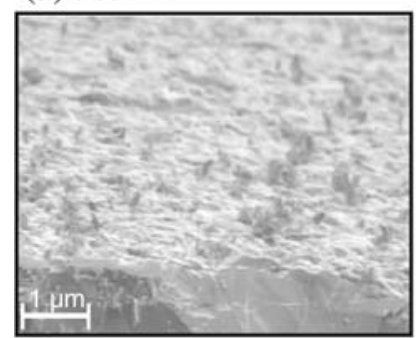

(c) $0.4 \mathrm{sccm}$ TMG

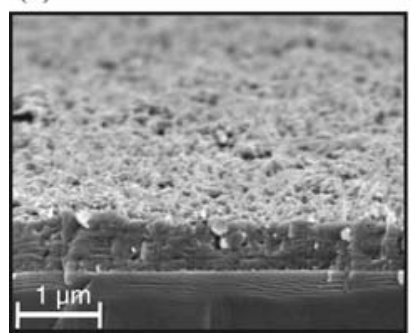

Fig. 4. SEM images of ZnO:Ga films grown on GaN(0001) templates, showing the effect of $\mathrm{Ga}$ doping on film and surface morphology. Ga has a profound and negative impact on morphology. (a) ZnO:Ga, TMG flow $=0.06 \mathrm{sccm}$; surface extremely rough, granular morphology. (b) ZnO:Ga, TMG flow = $0.1 \mathrm{sccm}$; columnar screw-like morphology. (c) $\mathrm{ZnO}: \mathrm{Ga}, \mathrm{TMG}$ flow $=0.4 \mathrm{sccm}$; layer is full of holes, granular.

3c-e shows samples with TMIn flows of $6 \mathrm{sccm}$, $12 \mathrm{sccm}$, and $25 \mathrm{sccm}$, respectively. In the overdoped regime, the morphology degraded, as hexagonal pits and hillocks were observed. One very important feature of all the In-doped films was that the film remained homogeneous and never displayed a columnar structure. A companion doping study using $\mathrm{Ga}$ as the $n$-type dopant consistently yielded $\mathrm{ZnO}$ films with columnar microstructure.

Experiments with $\mathrm{Ga}$ doping were performed prior to the In-doping study, using the same growth conditions, but with the injection of trimethylgallium (TMG) in the range of $0.06 \mathrm{sccm}$ to $0.4 \mathrm{sccm}$, which is in the heavy doping range. For this range of TMG flows, the carrier concentration of the films did not increase. The sample grown with $0.06 \mathrm{sccm}$ TMG ([Ga] $10^{21} \mathrm{~cm}^{-3}$ as measured by SIMS) was the only one to which we were able to contact successfully. It had a carrier concentration of $8.8 \times 10^{16} \mathrm{~cm}^{-3}$, which corresponds to the unintentional background doping. The sheet resistance and mobility of this sample were $1.2 \times 10^{4} \Omega /$ sq and $20 \mathrm{~cm}^{2} / \mathrm{Vs}$, respectively.

Figure 4 shows SEM images of the Ga-doped $\mathrm{ZnO}$ layers grown in this study. It is clear that $\mathrm{Ga}$, even at such low injection levels, had a profound and deleterious impact on film morphology, as these layers were granular, extremely rough, and the sample shown in Fig. 4b had a columnar screw-like morphology.

We performed an experimental study on In doping of $\mathrm{ZnO}(0001)$ films on $\mathrm{GaN}(0001)$ templates. The peak carrier concentration realized was $1.82 \times$ $10^{19} \mathrm{~cm}^{-3}$, and a minimum sheet resistance of $184 \Omega / \mathrm{sq}$ was achieved. Indium is an effective dopant for controlling the $n$-type conductivity of $\mathrm{ZnO}$ grown on $\mathrm{GaN}(0001)$ templates, and it is now of interest to implement these films as contacts to nitride-based devices. We are conducting studies to understand the cause of the microstructural degradation of the $\mathrm{ZnO}$ :In films, investigating the optical properties of these films, and developing highquality In-doped $\mathrm{ZnO}$ contacts to $n$-type and $p$-type GaN. Also, we are exploring additional film deposition conditions and growth structures to further drive up the conductivity of the $\mathrm{ZnO}$ :In films so that they can compete with ITO films. For example, we are investigating how varying growth temperature and VI/II ratio can promote indium incorporation. We are also growing $\mathrm{ZnO}$ :In films with In-doped buffer layers rather than UID buffers and are also directly depositing epitaxial films without the use of a buffer layer, with the goal of further increasing the conductivity of the In-doped $\mathrm{ZnO}$ films.

\section{ACKNOWLEDGEMENTS}

This work was supported by MRSEC Program of the National Science Foundation under Award No. DMR05-20415 and by SSLEC.

\section{OPEN ACCESS}

This article is distributed under the terms of the Creative Commons Attribution Noncommercial License which permits any noncommercial use, distribution, and reproduction in any medium, provided the original author(s) and source are credited.

\section{REFERENCES}

1. W.H. Hirschwald, Acc. Chem. Res. 18, 228 (1985).

2. U. Özgür, Y.I. Alivov, and C. Liu, J. Appl. Phys. 98, 4130 (2005).

3. S.J. Pearton, C.R. Abernathy, and M.E. Overberg, J. Appl. Phys. 93, 1 (2003).

4. D.G. Thomas, J. Phys. Chem. Solids 15, 86 (1960).

5. A. Janotti and C.G. Van de Walle, Appl. Phys. Lett. 87, $122102(2005)$.

6. D.C. Look, J.W. Hemsky, and F.R. Sizelove, Phys. Rev. Lett. 82, 2552 (1999).

7. C.G. Van de Walle, Phys. Rev. Lett. 85, 1013 (2000).

8. V. Bhosle, A. Tiwari, and J. Narayan, Appl. Phys. Lett. 88, 032106 (2006).

9. W. Sato, Y. Itsuki, and S. Morimoto, Phys. Rev. B 78, 045219 (2008).

10. K. Tamura, K. Nakahara, and M. Sakai, Phys. Status Solidi 201, 2704 (2004).

11. T. Ive, T. Ben-Yaacov, A. Murai, H. Asamizu, and J.S. Speck, Phys. Status Solidi 5, 3091 (2008).

12. K. Nakahara, Jpn. J. Appl. Phys. 43, L180 (2004).

13. T. Margalith, O. Buchinsky, and L.A. Coldren, Appl. Phys. Lett. 74, 3930 (1999).

14. C. Guillen and J. Herrero, J. Appl. Phys. 101, 073514 (2007).

15. L. Kerkache, A. Layadi, E. Dogheche, and D. Remiens, Eur. Phys. J. Appl. Phys. 39, 1 (2007).

16. UCSB unpublished results.

17. M.A.L. Johnson, S. Fujita, and W.H. Rowland, J. Electron. Mater. 25, 855 (1996).

18. H.J. Ko, Y.F. Chen, S.K. Hong, H. Wenisch, T. Yao, and D.C. Look, Appl. Phys. Lett. 77, 3761 (2000).

19. T. Ive, T. Ben-Yaacov, and J. Speck, J. Cryst. Growth 310, 3407 (2008). 